\title{
Application of Continuous and Structural ARMA Modeling for Noise Analyses of a BWR Coupled Core and Plant Instability Event
}

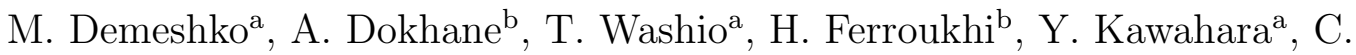 \\ Aguirre $^{\mathrm{c}}$ \\ ${ }^{a}$ The Institute of Scientific and Industrial Research, Osaka University, 8-1, Mihogaoka, \\ Ibaraki City, Osaka, 567-0047, Japan \\ ${ }^{b}$ Paul Scherrer Institut, 5232 Villigen PSI,Switzerland \\ ${ }^{c}$ Kernkraftwerk Leibstadt 5235 Leibstadt, Switzerland
}

\begin{abstract}
This paper presents a first application of a novel Continuous and Structural Autoregressive Moving Average (CSARMA) modeling approach to BWR noise analysis. The CSARMA approach derives a unique representation of the system dynamics by more robust and reliable canonical models as basis for signal analysis in general and for reactor diagnostics in particular. In this paper, a stability event that occurred in a Swiss BWR plant during power ascension phase is analyzed as well as the time periods that preceded and followed the event. Focusing only on qualitative trends at this stage, the obtained results clearly indicate a different dynamical state during the unstable event compared to the two other stable periods. Also, they could be interpreted as pointing out a disturbance in the pressure control system as primary cause for the event. To benchmark these findings, the frequencydomain based Signal Transmission-Path (STP) method is also applied. And with the STP method, we obtained similar relationships as mentioned above. This consistency between both methods can be considered as being a confirmation that the event was caused by a pressure control system disturbance
\end{abstract}

Email addresses: marina@ar.sanken.osaka-u.ac.jp (M. Demeshko), abdelhamid.dokhane@psi.ch (A. Dokhane), washio@ar.sanken.osaka-u.ac.jp (T. Washio), hakim.ferroukhi@psi.ch (H. Ferroukhi), kawahara@ar.sanken.osaka-u.ac.jp (Y. Kawahara), carlos.aguirre@kkl.ch (C. Aguirre)

Preprint submitted to Annals of Nuclear Energy

August 18, 2014

(C) 2014. This manuscript version is made available under the Elsevier user license

http://www.elsevier.com/open-access/userlicense/1.0/ 
and not induced by the core. Also, it is worth noting that the STP analysis failed to catch the relations among the processes during the stable periods, that were clearly indicated by the CSARMA method, since the last uses more precise models as basis.

Keywords: MultiVariate AR/ARMA Modeling, Canonical Modeling, Discrete and Continuous Time Signals, Reactor Noise and Causality Analysis, BWR stability and dynamics

\section{Introduction}

The development and/or application of time-series-analysis and system identification techniques dedicated to instrumentation, monitoring and diagnostics of Light-Water-Reactor (LWR) processes or for inference of physical mechanisms from the latter, has been and continues to be a matter of intensive research (Williams, 1977; Fry et al., 1984; Sweeney, 1987; Pazsit, 1999; Zylbersztejn et al., 2013). For Boiling-Water-Reactors (BWRs), a specific and important target application of time-series-analysis that can be mentioned is the evaluation of the reactor stability properties using measured neutron flux signals as basis.

The BWR constitutes indeed a dynamical system and can as such be considered as stable when returning to an asymptotically equilibrium condition after that an initial departure from this condition has occurred due to some disturbances. But in this context, one must distinguish between core stability, which refers to fluctuations in coolant flow and power generation process coupled via nuclear feedback, and plant stability which instead refers to interactions of control and mechanical systems (e.g., reactor pressure, feedwater flow and pump) with processes occurring in the core. To evaluate the core stability properties, system identification methods based on univariate AutoRegressive Moving-Average (ARMA) modeling of neutron flux signals are usually applied (Rotander, 1999). This is for instance the case in the PSI methodology developed over the years for applications to the Swiss reactors (Dokhane et al., 2006). Typically, the ARMA models are estimated based on the assumption that process noise (e.g., pressure and temperatures) excites the core dynamics and that the driving noise is white. The advantage is that the ARMA models can then be used in a black-box manner when estimating the core stability properties, namely the decay ratio (DR) and the resonance frequency $(\mathrm{RF})$, even if model parameters have no physical correspondence. 
Now regarding oscillatory modes related to plant stability, these can in most case be clearly separated from the core stability mode. However, interactions between the two could, under certain circumstances, take place and in this case, it might no longer be straightforward to identify the noise source that is driving the fluctuations and to establish thus a cause and effect relationship (Kwatny and Fink, 1975; Wilson, 2006; Kanemoto et al., 1982).

This was precisely the case for an event that occurred in recent years in a Swiss BWR plant, Kernkraftwerk Leibstadt (KKL) (Ferroukhi, 2008). It was found that this event was caused by intense interactions between undamped neutron flux oscillations and specific process signal fluctuations but without revealing the causality relationship.

To address this, causality analysis methods based on multivariate or Vector Autoregressive (VAR) and Autoregressive Moving-Average(VARMA) models become necessary (Ferroukhi, 2008; Oguma, 1981). The parameters of such VAR/VARMA model do not need to have any physical correspondence. Also, these VAR/VARMA models rely on discreet-time representations of the system dynamics. Moreover, a VARMA model is not canonical, i.e., it does not uniquely represent the objective reactor processes, since a given time series can be generated by the infinite number of the VARMA models as we explain later (Gottschalk, 2001; Moneta et al., 2010; Demeshko et al., 2014). This limitation makes it difficult to understand the dynamics of the objective system and its individual processes.

One of the widely used causality analysis methods based on multivariate time series modeling is the Signal Transmission Path (STP) analysis (Oguma, 1981, 1982a,b; Oguma and Turkan, 1985; Oguma, 1996). However, this method is carried out based on the non-canonical VARMA model. Therefore, the STP analysis has a limitation similar to VARMA modeling and its results can have some discrepancy with the causal structure of the actual nuclear plant.

In this paper, we apply a novel VARMA modeling method developed at Osaka University, called Continuous and Structural Autoregressive Moving Average (CSARMA) approach (Demeshko et al., 2013, 2014), that is precisely aimed to overcome this limitation. Based on the generic belief that the system can be represented by a combination of multiple individual processes, this approach derives canonical models of the systems dynamics. The models uniquely represent the system and each equation in the models has bijective correspondence to each individual process in the system. These canonical models, called Structural Vector Autoregressive Moving Average 
(SVARMA) model and Continuous Time Vector Autoregressive Moving Average (CVARMA) model, allow us to investigate the dependency among the processes in the system and give us more insights on the physical mechanisms of the system (Moneta et al., 2010; Stamer et al., 1996). Through this, the canonical models matrices should then indicate physical relationships between the signals and this could in turn allow to study underlying interactions and/or gain deeper insight into physical mechanisms of the system dynamics.

So far the verification of the CSARMA method has been limited to numerical cases and it therefore was considered valuable to attempt assessing its applicability for reactor noise analysis in general and for causality analysis in particular. To that aim, the KKL plant instability event was considered as appropriate situation target. And since the CSARMA approach is based on more reliable models, we expect to gain deeper insight into physical mechanisms of the system dynamics. To benchmark the results of the CSARMA method, the frequency-domain based STP method, currently being implemented at PSI, was also applied.

The scope of the work presented in the following sections. Section 2 gives the description of analyzed KKL plant instability event. Section 3 explains the principles of the CSARMA modeling applied in our work. The results of the CSARMA application to the KKL instability event and their interpretation are presented in section 4 . Section 5 describes the STP analysis and its results. In section 6 we discuss the differences and the agreements of the results of the CSARMA and the STP approaches. Section 7 presents a summary of the analyses in this study and the future plan for research.

\section{KKL plant instability event}

\subsection{Description}

During start-up of a 24th KKL cycle, high decay ratios (DR) with a corresponding large resonance frequency $(\mathrm{RF})$ were suddenly indicated by the plant on-line stability monitoring system. During that time, the reactor was in the power ascension phase with the specific objective to raise the thermal power from $18 \%$ to $27 \%$ while maintaining a core flow around $34 \%^{1}$. The time evolution of some main process signals preceding, during and following the event is illustrated in Fig. 1. For simplicity, the time-frame has been

\footnotetext{
${ }^{1} 100 \%$ power $=3600 \mathrm{MW}, 100 \%$ flow $=11151 \mathrm{~kg} / \mathrm{s}$
} 


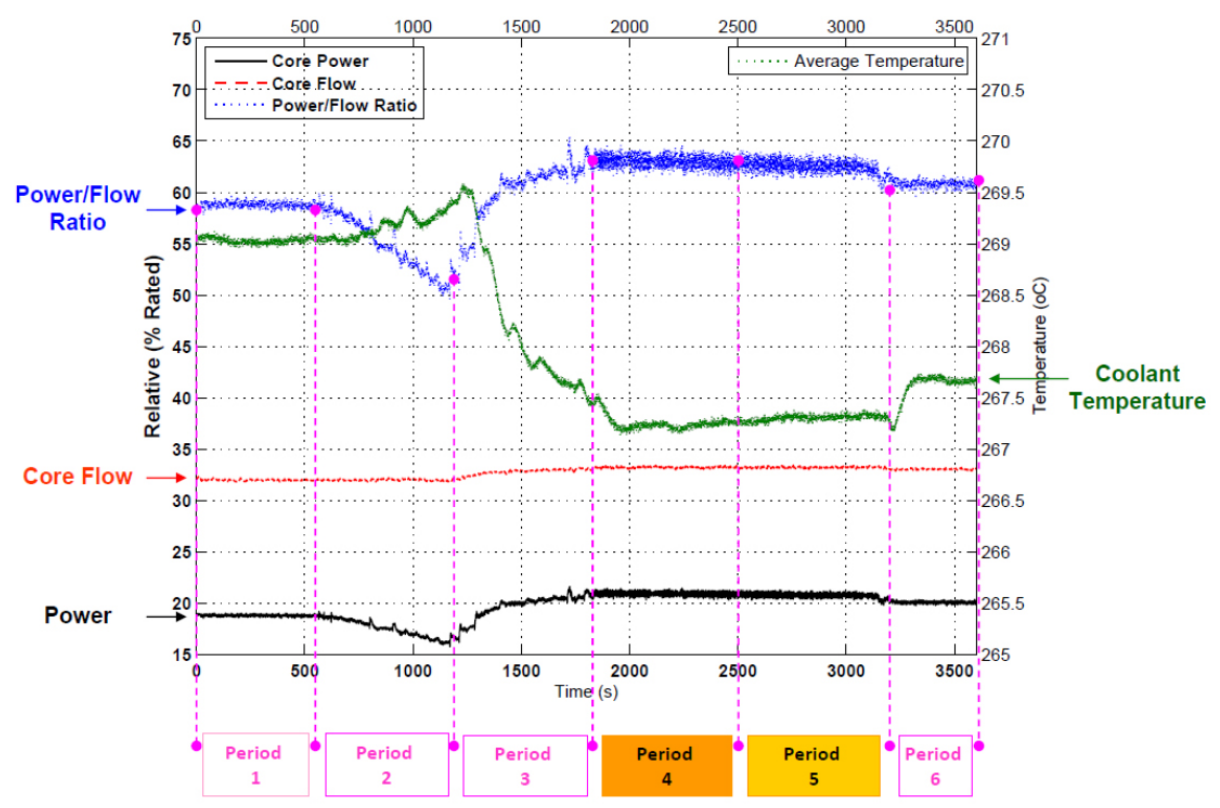

Figure 1: Time Evolution of Main process Parameters during KKL Plant Instability Event.

decomposed into 6 periods with the main characteristics summarized in Table 2.1.1. The core was thus indicated as having become unstable during periods 4 and 5 each of which lasted around 10 minutes. However, during the entire time frame, the power/flow ratio $(\mathrm{P} / \mathrm{F})$ remained below 0.65 which is clearly very low compared to the $\mathrm{P} / \mathrm{F}$ ratio range where core instability mechanisms might potentially be triggered.

\subsection{Time Series Analysis}

Because of the unexpected occurrence of undamped neutron flux oscillations in this operating range, a time-series-analysis of available measured signals was conducted shortly after the event (Ferroukhi, 2008). On the one hand, one objective was to verify if the instability alarms were triggered by erroneous evaluations of the core stability properties by the on-line stability monitoring system. The reason is that the latter employed a non-parametric method based on autocorrelation functions (ACF) which do not contain any phase information. Thereby, in case of several underlying oscillations at different frequencies, the ACF results might be associated with high uncertainties depending on the relative differences in power spectral density of the various oscillation components. Therefore, the PSI methodology based 
Table 1: Characteristics of Time Periods during KKL Plant Instability Event

\begin{tabular}{cllc}
\hline $\begin{array}{c}\text { PeriodProcess } \\
\text { Characteristics }\end{array}$ & Reactor Manoeuvres & $\begin{array}{c}\text { On-Line } \\
\text { Stability } \\
\text { Monitoring }\end{array}$ \\
\hline \hline 1 & Stationary & - & Stable \\
\hline 2 & Non-Stationary & $\begin{array}{l}\text { Power Reduction } \\
\text { (CR insertion and reduced Subcooling) }\end{array}$ & Stable \\
\hline 3 & Non-Stationary & $\begin{array}{l}\text { Power Increase } \\
\text { (Feedwater Injection followed by }\end{array}$ & Stable \\
& & CR withdrawal) & \\
\hline 4 & Stationary & - & Unstable \\
\hline 5 & Stationary & - & Unstable \\
\hline 6 & Stationary & $\begin{array}{l}\text { None but following a short power reduction } \\
\text { (CR Movements and reduced Subcooling) }\end{array}$ & Stable \\
\hline \hline
\end{tabular}

on ARMA model identification from measured neutron flux signals (Wilson, 2006) was applied and the results are illustrated in Fig. 2, noting that results shown there were estimated based on a weighting of the underlying dominant oscillation frequencies upon their associated power spectral density. Clearly, this analysis confirmed the correctness of the on-line stability indications with a jump in frequency up to $0.9 \mathrm{~Hz}$ accompanied by a decay ratio close to 1.0 .

To understand the reasons for the above behavior, the time-series-analysis was enlarged to a complete signal analysis of available measured signals including thus spectral, phase and coherence analyses. For simplicity, results will hereinafter only be reported for Periods 1 and 6 to represent time-frames for stable conditions prior and after the event respectively. And only results for Period 4 will be reported as representative of the unstable event time frames. Hence, for the stable Periods $1 / 6$ and the unstable period 4 , the power spectral density of neuron flux and selected process signals are shown in Fig. 3. Clearly during Period 4, a strong spectral peak previously not present appeared at around $0.9-1 \mathrm{~Hz}$ in all the signals with marquantly high spectral density especially in the steam flow signals. Once the core went back to indicated stable conditions (Period 6), such peak remained in the steam flow and neutron flux although with much lower power density and basically 


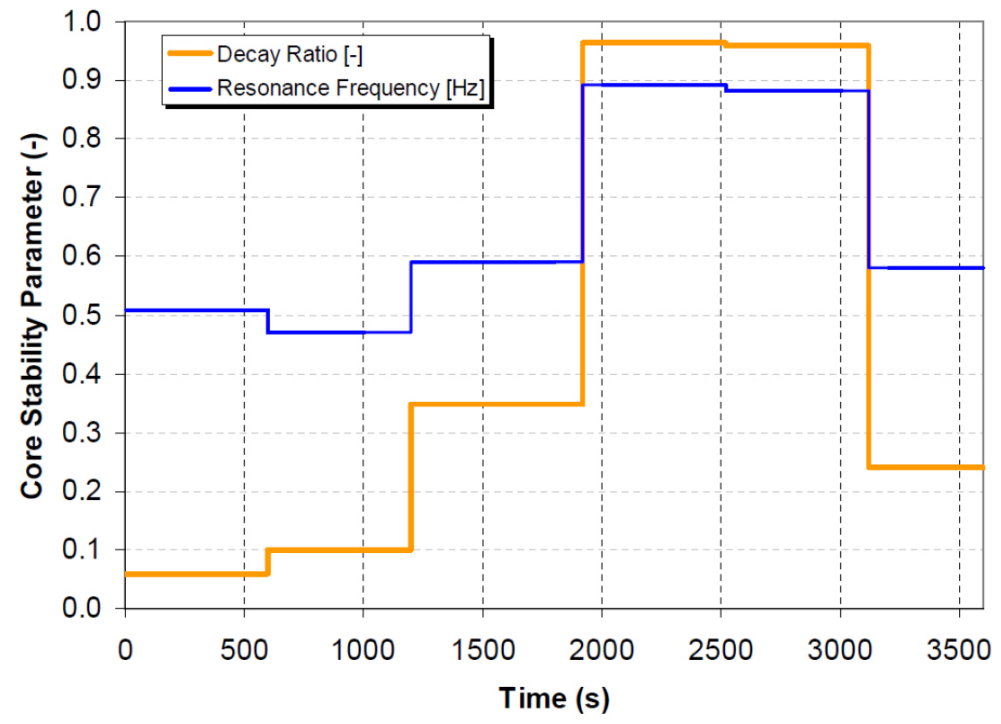

Figure 2: PSI Evaluation of Decay Ratio and Resonance Frequency during stability Event.

disappeared from the reactor pressure spectrum

To further investigate the observed peaks, phase- and coherence analyses between all signals were for all periods conducted. For Period 4, illustrative results of the coherence analysis between neutron flux and steam flow are illustrated in Fig. 4. There, corresponding results from a stability test carried out at the plant and during which the core was brought close to unstable conditions, are also shown. For this test conducted at very low flow conditions, the measured decay ratio and resonance frequency evaluated from neutron noise signals were 0.97 and $0.47 \mathrm{~Hz}$ respectively.

From Fig. 4, the first observation is a full and $\mathrm{COH}$ between neutron flux and steam flow around 0.9-1 Hz. The same trend was observed between neutron fluxes and all other signals as well as between the latter. Thereby, it appeared clear that the undamped neutron flux oscillations causing the instability alarm were clearly the result of interactions with process signals related to pressure control (e.g., via steam flow) and/or eventually feedwater control. As causality analysis methods were not implemented at the time of this time-series-analysis, the comparison with the stability test served the purpose of providing indications of the relationship between (core) neutron flux and (plant) steam flow. Because in this case, it was known that the core was close to unstable without any interactions from plant control systems. 

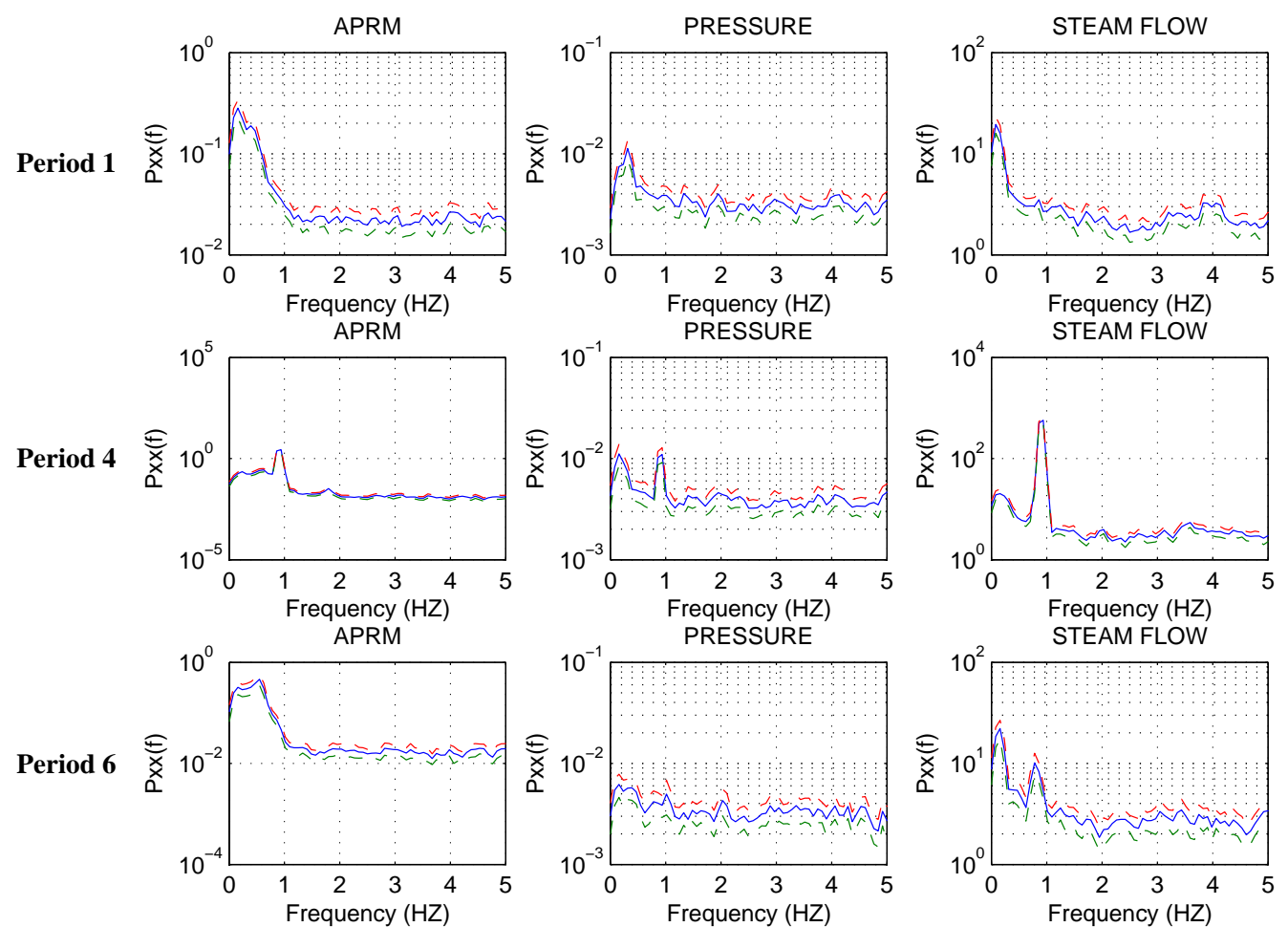

Figure 3: Spectral Analysis of Selected Measured Neutron- and Process Signals.

This is indeed reflected by a very distinct large neutron flux spectral peak at around $0.5 \mathrm{~Hz}$, i.e., in the expected core natural frequency range while the stream flow only shows a slightly damped peak above this frequency, something that could to some extent be indicative of a shift due to the time constants between fission power oscillations and resulting steam outflow response. However, the main observation deduced from the practically zero $\mathrm{COH}$ was that in case of an unstable core with strong neutron flux oscillations, this would not produce corresponding strong oscillations of the steam flow. Thereby, it was concluded that most likely, the start-up stability event was driven by noise disturbances and fluctuations stemming from interactions with plant control systems. Recently, work towards confirming this by causality analysis methods was initiated and this is thus presented in the flowing sections. 

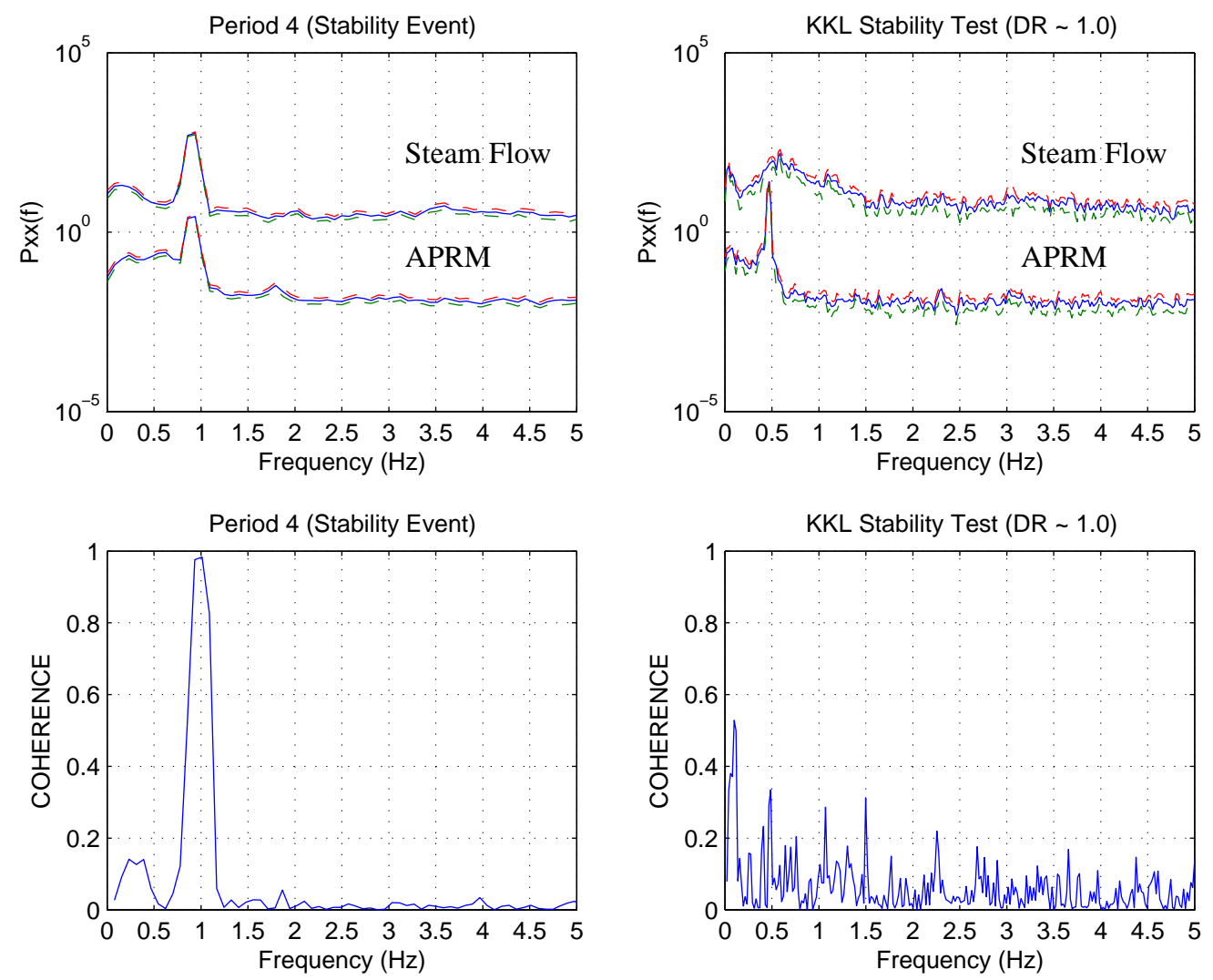

Figure 4: Comparison of Spectral- and Coherence Analysis for Neutron Flux and Steam Flow between KKL Plant Instability Event and Stability Test with close to Unstable Core.

\section{CSARMA method}

For understanding the physical mechanisms of the reactor dynamics, we need to have a mathematical model and its corresponding parameters that precisely reflect the reactor processes. Since the reactor noise generation processes can be often approximated by a linear Markov system we apply ARMA modeling to obtain the model and the parameters in the framework of the parametric analysis. However, the univariate methods do not provide sufficiently detailed information on the mechanisms of reactor dynamics, since they cannot analyze influences among multiple processes in the system. In contrast, the multivariate or Vector AR/ARMA modeling is widely used to 
derive the information on the influences among the processes in both time and frequency domains. Thus, for causality analysis we apply VARMA modeling.

\subsection{VARMA modeling}

The VARMA model shown in Eq.(1) is widely used for the parametric reactor stability analyses. It is a general representation of a multivariate linear Markov system in discrete time domain (Brockwell and Davis, 1991), which is a discrete time parametric approximation of the multivariate continuous time linear Markov system.

$$
Y(t)=\sum_{j=1}^{p} \Phi_{j} Y(t-j \Delta t)+U(t)+\sum_{j=1}^{q} \Theta_{j} U(t-j \Delta t)
$$

where $\Phi_{j}$ and $\Theta_{j}$ are $d \times d$ autoregressive (AR) and moving average (MA) coefficient matrices, respectively. $Y(t)$ and $U(t)$ are $d$-dimensional vectors of observed variables and unobserved noise, respectively. $\mathrm{p}$ and $\mathrm{q}$ are AR and MA model orders.

The primary principle used for the estimation of the VARMA model parameters is based on the maximum likelihood estimation. Let $\Theta=\left(c, \Phi_{1} \ldots \Phi_{p}\right.$, $\left.\Theta_{1}, \ldots, \Theta_{q}, \sigma^{2}\right)^{\prime}$ denote the vector of population parameters. Suppose we have observed a sample of size $T\left(y_{1}, y_{2}, \ldots, y_{T}\right)$. The approach will be to calculate the joint probability density

$$
f_{Y_{T}, Y_{T-1}, \ldots, Y_{1}}\left(y_{T}, y_{T-1}, \ldots, y_{1} ; \Theta\right)
$$

which might loosely be viewed as the probability of having these particular observed samples. The maximum likelihood estimate of $\Theta$ is the value for which this sample is most likely to have been observed; that is, it is the value of $\Theta$ that maximizes Eq. (2) (Shea, 1987).

\subsection{Canonical VARMA modeling}

Here, we point out that the VARMA model has some ambiguity to represent the system. Under any $d \times d$ regular matrix $Q$, Eq.(1) is transformed to the following equivalent representation.

$$
Y(t)=\sum_{j=1}^{p} \Phi_{j} Y(t-j \Delta t)+Q W(t)+\sum_{j=1}^{q} \Theta_{j} Q W(t-j \Delta t),
$$


where $W(t)=Q^{-1} U(t)$. As noted in the first section, assuming that the objective system can be represented by a combination of multiple individual processes, the model is canonical when the model uniquely represents the system where each equation in the model has bijective correspondence to each individual process in the system. However, we see that $Y(t)$ has infinitely various impulse responses for $W(t)$, depending on the choice of $Q$ (Moneta et al., 2010). Thus, the VARMA model is not a unique representation of the system. In other words, the VARMA model is not canonical.

This non-canonicality of the VARMA model comes from the lack of information on the very fast process dynamics of the objective system, i.e., continuous process dynamics that evolves within the sampling time period $\Delta t$. Equation (3) can be easily rewritten as follows:

$$
Y(t)=\sum_{j=0}^{p} \Psi_{j} Y(t-j \Delta t)+W(t)+\sum_{j=1}^{q} \Omega_{j} W(t-j \Delta t),
$$

where $\Psi_{0}=I-Q^{-1}, \Psi_{j}=Q^{-1} \Phi_{j}$ and $\Omega_{j}=Q^{-1} \Theta_{j} Q$ (Kawahara et al., 2011). This equation explicitly represents the very fast process dynamics including a feedback effect of $Y(t)$ on itself by the matrix $\Psi_{0}$. We see that the matrix $\Psi_{0}$ is provided by the continuous process dynamics evolving within the sampling time period $\Delta t$. If such very fast process dynamics is not included in the objective system, $\Psi_{0}$ must be zero, otherwise $\Psi_{0}$ is non-zero and thus $Q \neq I$. Accordingly, if we derive a unique $\Psi_{0}$ reflecting the very fast dynamics of the system, the unique combination of $Q$ and $W(t)$ in Eq.(4) representing the objective system is given, and it further provides a unique VARMA model where each equation and each element of the external noise bijectively correspond to an individual process in the system. This model is canonical and considered to more precisely represent the dependency structure among the observed variables. Hence, it is called a Structural Vector Autoregressive Moving Average (SVARMA) model (Moneta et al., 2010; Kawahara et al., 2011).

The most important issue in the derivation of the SVARMA model is how to derive the unique $\Psi_{0}$ (or $Q$ ) under the lack of the information on the very fast dynamics of the system. To fill the information, past studies on derivation of SVARMA models used specific domain knowledge on the system, such as the acyclicity of the influences among the system's processes and the non-Gaussianity of its external noises of the variables (Kawahara et al., 2011), and system representation by a small number of parameters and 
partial independence of the noises (Mainassara and Francq, 2009). However, such domain knowledge is not readily available or applicable for the reactor stability analysis in many cases.

Besides, when the system is multivariate, continuous time and linear Markov, it can be described by a generic Continuous time Vector Autoregressive Moving-Average (CVARMA) model.

$$
Y^{(p)}(t)=\sum_{m=0}^{p-1} S_{m} Y^{(m)}(t)+W^{(0)}(t)+\sum_{m=1}^{q} R_{m} W^{(m)}(t),
$$

where $Y^{(m)}(t)$ and $W^{(m)}(t)$ are the m-th time derivatives of $Y(t)\left(=Y^{(0)}(t)\right)$ and $W(t)\left(=W^{(0)}(t)\right)$, and $S_{m}$ and $R_{m}$ are $d \times d$ autoregressive (AR) and moving average (MA) coefficient matrices, respectively. A CVARMA model shown in Eq.(5) is known to be canonical (Brockwell and Davis, 1991; Stamer et al., 1996) by the following reason. Equation (5) is rewritten by introducing some regular matrix $P$ and a new noise vector $E(t)$ as follows, similarly to the SVARMA model.

$$
Y^{(p)}(t)=A Y^{(p)}(t)+\sum_{m=0}^{p-1} S_{m}^{\prime} Y^{(m)}(t)+E^{(0)}(t)+\sum_{m=1}^{q} R_{m}^{\prime} E^{(m)}(t),
$$

where $E(t)=P^{-1} W(t), A=I-P^{-1}, S_{m}^{\prime}=P^{-1} S_{m}$ and $R_{m}^{\prime}=P^{-1} R_{m} P$. Because the CVARMA model is an exact representation of the continuous processes in the objective system and thus includes all process dynamics, any extra dynamics governing $Y^{(p)}(t)$ does not exist. Thus, the matrix $A$ is zero, and matrix $P$ is equal to an identity matrix. Therefore, there exists only one combination of $P$ and $W(t)$ representing the objective system that provides a unique CVARMA model. In other words, the CVARMA model is canonical.

The CSARMA modeling approach (Demeshko et al., 2013, 2014) used in this study allows us to derive a SVARMA and also a CVARMA models of the objective system from its given VARMA model when the objective continuous time linear Markov system is stationary, controllable and observable. In the following chapter, we explain the CSARMA approach.

\subsection{Principle of the CSARMA method}

The CSARMA modeling approach to derive the SVARMA and the CVARMA models requires generic assumptions of stationarity, controllability and observability of the objective system which do not virtually limit its applicability. The system is stationary if mean value and standard deviation of the 
system state do not change with time. The system is observable, if the current state of the system can be determined by using its past observations over a finite time period, and the system is controllable if its current state can be transfered to any given state by applying an appropriate input series over a finite time period. Since these three conditions are required by the standard VARMA modeling, the CSARMA modeling approach doesn't introduce any extra assumptions to the system.

These assumptions are generic and applicable to reactor noise analysis. We can approximate the early stage of the reactor instability by a stationary system, since the reactor is still within the stable region in many cases including the event KKL C24. Also, the reactor is designed in such a way that the correct estimation of its current state by existing monitoring systems and the derivation of its state to any given objective state by the control system are always possible under its normal condition. Hence, the normal reactor is always observable and controllable. Accordingly, all conditions required for the application of the CSARMA modeling are met for the reactor noise analysis.

The CSARMA modeling approach is based on the relations between the CVARMA, the SVARMA and the VARMA models. Since the CVARMA and the SVARMA models are both canonical, i.e., their equations have bijective correspondences to the processes in the objective system, they should bijectively correspond each other. Therefore, the SVARMA model is a discrete time approximation of the CVARMA model. By applying finite difference approximation scheme named backward Euler formula upto the m-th derivative (Levy and Lessman, 1992) to Eq.(5), the CVARMA model is explicitly related with the SVARMA model as follows (Demeshko et al., 2014).

$$
\begin{aligned}
& \Psi_{0}=I+\left(\sum_{m=0}^{q} R_{m} \Delta t^{p-m}\right)^{-1} \sum_{m=0}^{p} S_{m} \Delta t^{p-m}, \\
& \Psi_{j}=(-1)^{j}\left(\sum_{m=0}^{q} R_{m} \Delta t^{p-m}\right)^{-1} \sum_{m=j}^{p} \frac{m !}{(m-j) ! j !} S_{m} \Delta t^{p-m},
\end{aligned}
$$

where $1 \leq j \leq p$ and $S_{p}=-I$, and

$$
\Omega_{j}=(-1)^{j}\left(\sum_{m=0}^{q} R_{m} \Delta t^{p-m}\right)^{-1} \sum_{m=j}^{q} \frac{m !}{(m-j) ! j !} R_{m} \Delta t^{p-m}
$$


where $1 \leq j \leq q$ and $R_{0}=I$. Since both models bijectively correspond to each other, these relations are further transformed into the backwards relations deriving Autoregressive (AR) parameters of the CVARMA model from the given parameters of the SVARMA model as (Demeshko et al., 2014)

$$
\begin{aligned}
& S_{0}=\Delta t^{-p} I- \\
& \sum_{m=1}^{p-1}\left\{(-1)^{m}\left(I-\sum_{k=1}^{q}(-1)^{k} \sum_{j=k}^{q} \frac{j !}{(j-k) ! k !} \Omega_{j}\right)^{-1} \sum_{j=m}^{p-1} \frac{j !}{(j-m) ! m !} \Psi_{j}\right. \\
& \left.+(-1)^{p+m-1} \Delta t^{-p} \frac{p !}{(p-m) ! m !} I\right\}+(-1)^{p} \Delta t^{-p} \Psi_{p}^{-1}\left(I-\Psi_{0}\right), \\
& S_{m}= \\
& (-1)^{m} \Delta t^{m-p}\left\{\Delta t^{p}\left(I-\sum_{k=1}^{q}(-1)^{k} \sum_{j=k}^{q} \frac{j !}{(j-k) ! k !} \Omega_{j}\right)^{-1} \sum_{j=m}^{p-1} \frac{j !}{(j-m) ! m !} \Psi_{j}\right. \\
& \left.+(-1)^{p-1} \frac{p !}{(p-m) ! m !} I\right\},
\end{aligned}
$$

where $1 \leq m \leq p-1$, and Moving Average (MA) parameters as

$$
\begin{aligned}
& R_{m}= \\
& (-1)^{m} \Delta t^{m}\left(I-\sum_{k=1}^{q}(-1)^{k} \sum_{j=k}^{q} \frac{j !}{(j-k) ! k !} \Omega_{j}\right)^{-1} \sum_{j=m}^{q} \frac{j !}{(j-m) ! m !} \Omega_{j},
\end{aligned}
$$

where $1 \leq m \leq q$.

On the other hand, as shown in the previous section, by knowing the unique $\Psi_{0}$, the VARMA model bijectively corresponds to the SVARMA model as follows (Kawahara et al., 2011):

$$
\begin{aligned}
\Psi_{j} & =\left(I-\Psi_{0}\right) \Phi_{j}, \\
\Omega_{j} & =\left(I-\Psi_{0}\right) \Theta_{j}\left(I-\Psi_{0}\right)^{-1} .
\end{aligned}
$$

The substitution of Eq.(12) into Eq.(9) and Eq.(10) and their further substitution into Eq.(6) and Eq.(7) allows us to derive $\Psi_{0}$ from the parameter matrices of a given VARMA model as follows.

$$
\Psi_{0}=I-(-1)^{p} \Delta t^{-p} \Phi_{p}^{-1}\left(\sum_{j=1}^{q} \Theta_{j} \sum_{k=1}^{j}(-1)^{k} \frac{j !}{(j-k) ! k !}-I\right) .
$$


Therefore, in case of a Markov linear, stationary, observable and controllable system in continuous time domain, the system is represented by a unique VARMA model.

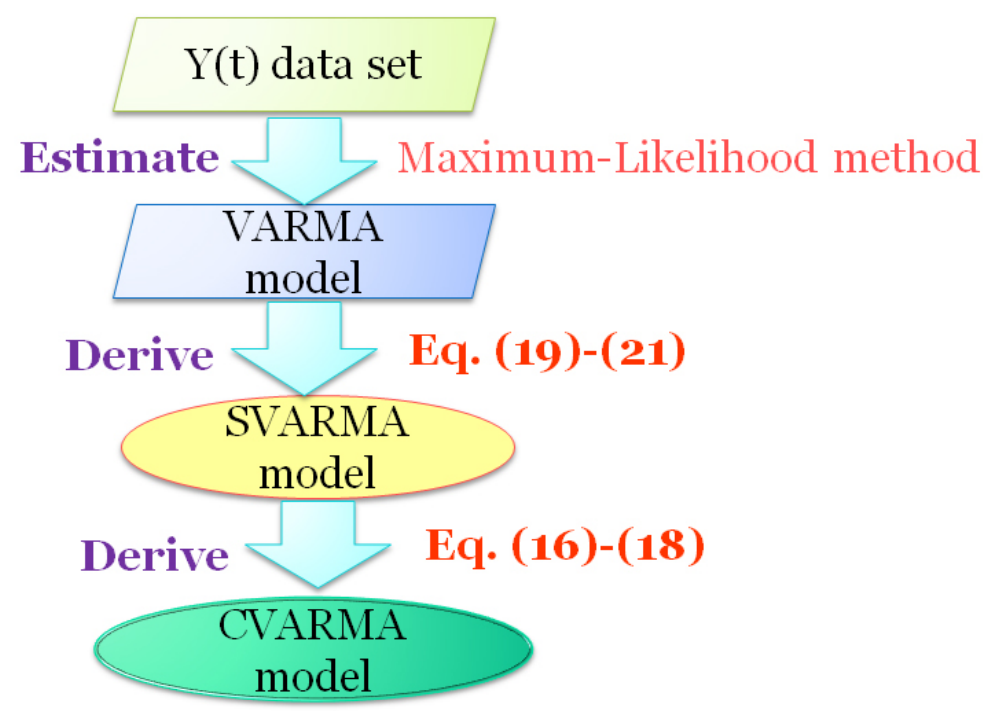

Figure 5: The scheme of the CSARMA algorithm.

Thus, the CSARMA modeling approach allows us to derive both the SVARMA and the CVARMA models of an objective system from the VARMA model which is estimated by using a given time series observed from the system. The scheme of the CSARMA algorithm is presented in Fig. 5. We firstly estimate a VARMA model by applying some traditionally used methods such as the Maximum Likelihood method which computes a likelihood function using a Kalman filter algorithm and applies a quasi-Newton algorithm to search for the maximum of the log-likelihood function (Shea, 1987). Then, we obtain a SVARMA model, Eq.(4), by deriving its parameter matrices from the matrices of the VARMA model through Eq.(12)-(14). The derived SVARMA model represents the physical dynamics of the system in canonical manner, and the parameter matrices $\Psi_{j}$ and $\Omega_{j}$ give us the information on the fundamental processes in the system. Finally, by using the relations between the SVARMA and the CVARMA models presented in Eq.(9)-(11), we estimate parameters of the CVARMA model of the system (Demeshko et al., 2014). 
For easy understanding of the effectiveness of the CSARMA modeling approach, we illustrate its application to a simple coupled oscillator in appendix A.

\section{Application of CSARMA for causality analysis of KKL Plant Instability Event}

For the analysis of the KKL stability analysis, particular intense effects between reactor pressure (PRESS), neutron fluxes (LPRM) and steam flow (SFLOW) were shown by the time series analysis. For these reasons, the CSARMA method is applied here for a 3-dimensional multi-variate system comprising $Y=[a=P R E S S, b=L P R M, c=S F L O W]^{t}$.

For the CSARMA application, it is necessary to know the model orders of the system. They were evaluated using time-series-analysis methodology developed over the years at Paul Scherrer Institute, using HPTSAC code, based on the combination of Akaike information criterion, Minimum description length principle, and the plateau method (Dokhane et al., 2006). According to the HPTSAC estimations, the dynamics of the objective processes are well represented by VARMA(4,3) model. Accordingly, for each period, we applied CSARMA method deriving CVARMA $(4,3)$ model from the estimated VARMA $(4,3)$ model, where the orders of the CVARMA model correspond to the orders of differential equations representing the system. The CVARMA AR matrices $S_{m}$ for $\mathrm{m}=0,1,2,3$ and MA matrices $R_{n}$ for $\mathrm{n}=1,2,3$ represent the dependency mechanisms among variables as follows,

$$
Z_{k}=\left(\begin{array}{ccc}
Z_{a->a} & Z_{b->a} & Z_{c->a} \\
Z_{a->b} & Z_{b->b} & Z_{c->b} \\
Z_{a->c} & Z_{b->c} & Z_{c->c}
\end{array}\right)
$$

, where $\mathrm{k}=\mathrm{m}$ if $Z_{k}$ is $\mathrm{AR}$ matrix $S_{m}$ and $\mathrm{k}=\mathrm{n}$ if $Z_{k}$ is MA matrix $R_{n}$, noting that

- each entry $Z_{X->Y}$ of the matrix is defined as the "signal pair interaction" from $\mathrm{X}$ to $\mathrm{Y}$;

- the absolute value $\left|Z_{X->Y}\right|$ is defined as the "interaction strength" between $\mathrm{X}$ to $\mathrm{Y}$;

- the sign of $Z_{X->Y}$ is defined as the "interaction direction" from $\mathrm{X}$ to $\mathrm{Y}$; 
- the model order represents time delays of order $\mathrm{K}^{*} \mathrm{~T}$ with $\mathrm{T}$ being the sampling period.

For periods 1,4 and 6 of KKL plant instability event, we first evaluated the ratios of the average value of the MA matrices over that of the AR matrices as follows:

$$
\varphi=\frac{\frac{1}{q} \sum_{k=1}^{q} \frac{1}{d^{2}} \sum_{i, j}\left|r_{k, i j}\right|}{\frac{1}{p} \sum_{k=1}^{p} \frac{1}{d^{2}} \sum_{i, j}\left|s_{k, i j}\right|},
$$

where $\left|r_{k, i j}\right|$ and $\left|s_{k, i j}\right|$ are absolute values of $i, j$-elements for $k$-order MA and AR matrices, respectively. The ratios are $0.14 \%, 0.18 \%$ and $0.19 \%$ for periods 1,4 and 6 , respectively. In addition, there are no exceptional large elements in any MA matrices that do not follow these ratios. Thus, we exclude the MA part of the CVARMA models in our analysis and concentrate on the AR part.

According to the above notation, the estimated diagonal elements of the CVARMA matrices represent the effect of a signal on itself, i.e., "self-effects". They are shown for the various signals, periods and matrices in Fig. 6, from which a few observations can be made. First, for each signal, the self-effects show a cyclic nature (change of sign) as function of matrix order, i.e., time shift. Secondly, for any matrix order, it is interesting to note a relatively much stronger self-effect for the steam flow compared to the two other signals. This might indicate that the steam flow is influenced by some external process not included in the three-signals system investigated here. That might be a disturbance in some control system or the influence of the feed water system, which can effect steam flow on such low power levels (Kwatny and Fink, 1975). However, these are just hypotheses and they need to be clarified in the future by additional investigation. Third, it is noted that the two stable Periods $1 / 6$ show a very similar behavior. However, also Period 4 show a similar pattern, the only minor difference being a reduction of the LPRM and steam-flow self-effects combined with an increase of the pressure selfeffect. However, overall, the patterns are rather similar between all periods indicating that the self-effects, i.e., the CVARMA diagonal terms, do not provide enough information to allow discriminating the core/plant dynamics between Periods $1 / 6$ and 4 .

Next, our focus is given to the effects between variables, i.e., the offdiagonal elements of the CVARMA matrices. The results of the CVARMA model excluding diagonal elements are shown in Fig. 7 noting that since qualitative trends are primarily aimed at, the $S_{X->Y}$ interaction is for each 


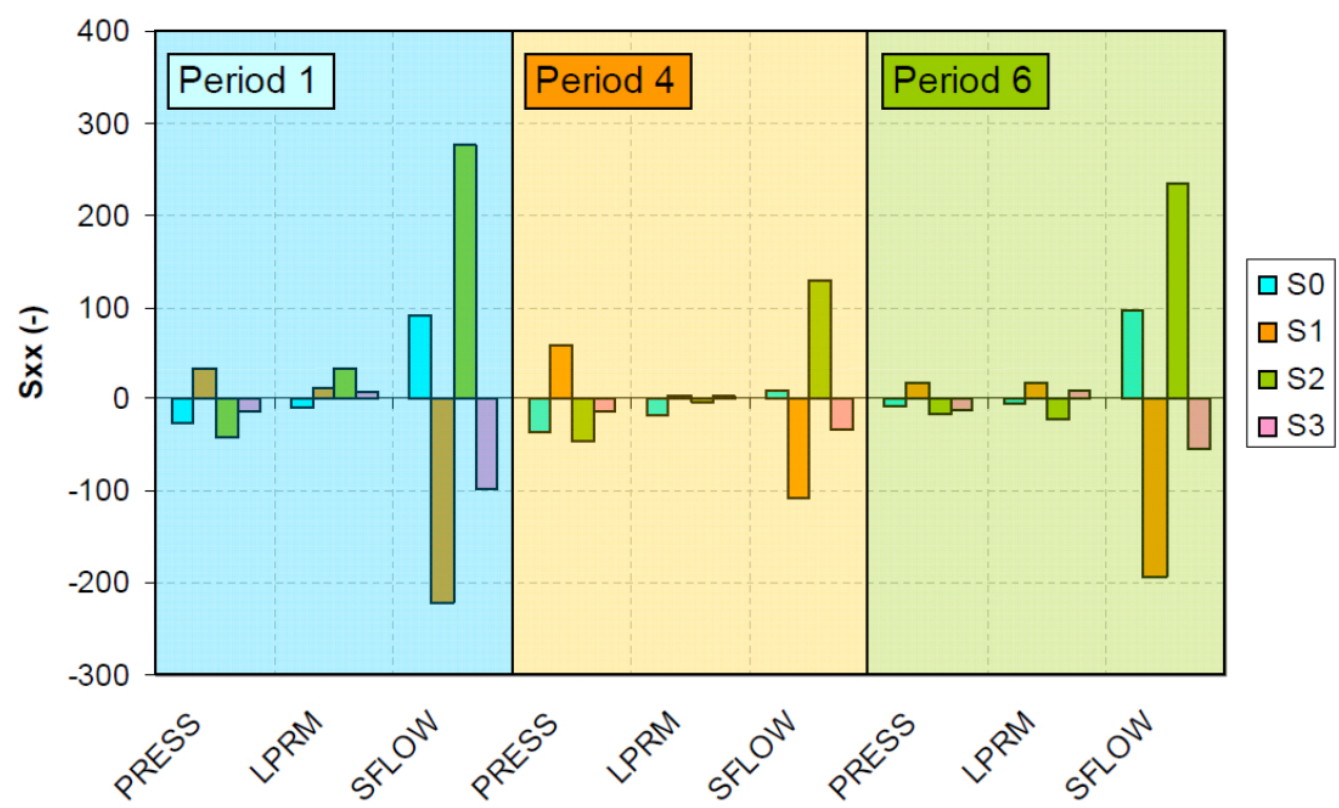

Figure 6: CSARMA Results for Self-Effects - Diagonal Matrix Elements.

signal pair normalized to average interaction strength between both signals, i.e.,

$$
\bar{S}_{X->Y}^{N o r m}=\frac{S_{X->Y}}{\left\langle\bar{S}_{X->Y}\right\rangle} \quad \forall \quad X \neq Y
$$

,where

$$
\left\{\left\langle\bar{S}_{X->Y}\right\rangle=\frac{1}{2}\left(\left|S_{X->Y}\right|+\left|S_{Y->X}\right|\right)\right\} .
$$

The following three major observations are obtained based on the qualitative features indicated by the CVARMA matrix off-diagonal elements.

- First, the causal structure is quite similar in the stable periods 1 and 6. This indicates that the core/plant dynamics represented by the CVARMA model returned to the similar dynamics after the instabilities occurred in period 4. 

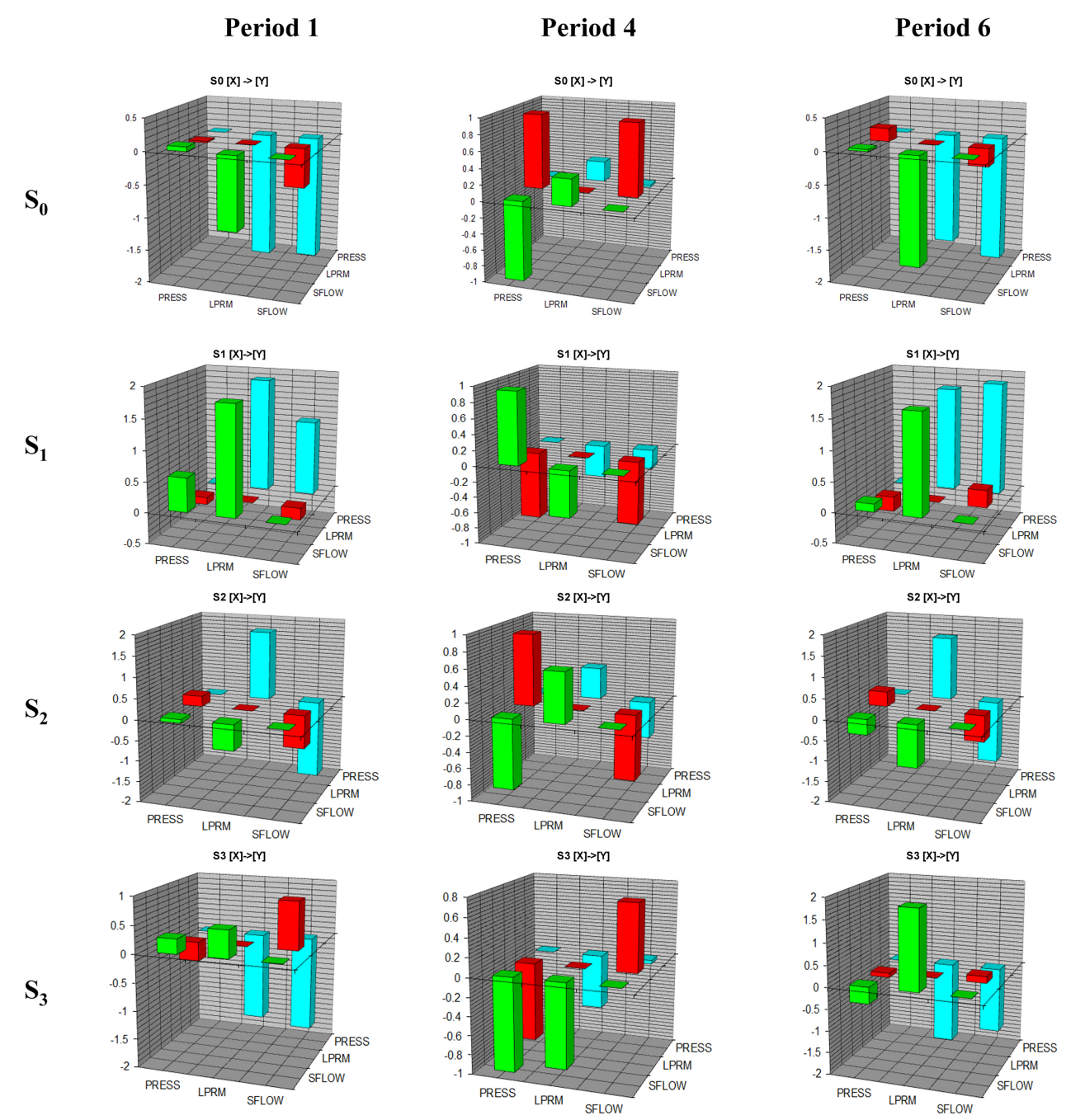

Figure 7: CSARMA results for casual structure - Off-Diagonal Elements, where the blue bars show the elements of the first row, the red bars - the elements of the second row, and the green bars - the elements of the third row of CVARMA models matrices.

- Secondly, the less stable period 4 shows on the other hand distinctly different patterns compared to the two other periods. This indicates that the CVARMA model has captured a complete change in the dynamical interactions between the three investigated variables during this period. And this is fully in-line with the fact that among all three 
periods, a core/plant related instabilities occurred indeed only during period 4 .

- Third, we note that the consistency between period $1 / 6$ and the distinctly difference of period 4 are observed in every matrix order, i.e., each time differential in the dynamics between the variables.

By closely looking at this figure, we notice that the dominant effects, $\{$ SFLOW $\Longrightarrow$ PRESS $\},\{L P R M \Longrightarrow$ PRESS $\}$ and $\{$ LPRM $\Longrightarrow$ SFLOW $\}$ are observed in some orders of the AR matrices at periods 1/6. For period 4 , a radically different behavior is seen. Indeed, the dominant effect is $\{$ PRESS $\Longrightarrow$ SFLOW $\}$ only, however we also observe some minor effects like $\{$ PRESS $\Longrightarrow$ LPRM $\},\{$ SFLOW $\Longrightarrow$ LPRM $\}$ and the feedback $\{$ SFLOW $\Longrightarrow$ PRESS $\}$ additionally. In other words, PRESS and SFLOW have bidirectional causality while the influence from PRESS is much bigger than other way around, which is radically differs from periods 1 and 6. Also the CSARMA results for period 4 indicate, it was $\{$ PRESS $\Longrightarrow$ SFLOW disturbances that induced/guided LPRM fluctuations and not the other way around. Finally, it is worth noting that the changes of the signs of elements show some oscillatory behavior over matrices orders for all the effects.

It was also noted earlier that the steam flow appeared to be exogenous and effected by some external source(s). Therefore, to verify the robustness of the results presented and discussed above, it was considered appropriate to verify the trends between $\{$ PRESS, SFLOW, LPRM $\}$ if an additional signal would be added to the multi-variate system analyzed with the CSARMA method. To that aim, a signal related to the feedwater control system and referred to here as SWP for simplicity, was selected since at low power/flow conditions, which were exactly the conditions prevailing during the event, lightly damped oscillations between pressure and feedwater control loops could potentially occur Kwatny and Fink (1975). On that basis, the following 4-dimensional multi-variate system was estimated: $Y=[q=S W P, a=P R E S S, b=$ $L P R M, c=S F L O W]^{t}$.

$$
S_{m}^{\prime}=\left(\begin{array}{cccc}
S_{q->q}^{\prime} & S_{a->q}^{\prime} & S_{b->q}^{\prime} & S_{c->q}^{\prime} \\
S_{q->a}^{\prime} & S_{a->a}^{\prime} & S_{b->a}^{\prime} & S_{c->a}^{\prime} \\
S_{q->b}^{\prime} & S_{a->b}^{\prime} & S_{b->b}^{\prime} & S_{c->b}^{\prime} \\
S_{q->c}^{\prime} & S_{a->c}^{\prime} & S_{b->c}^{\prime} & S_{c->c}^{\prime}
\end{array}\right) \quad(m=0,1,2,3)
$$

To verify the effect of using the above enlarged multi-variate system on the 


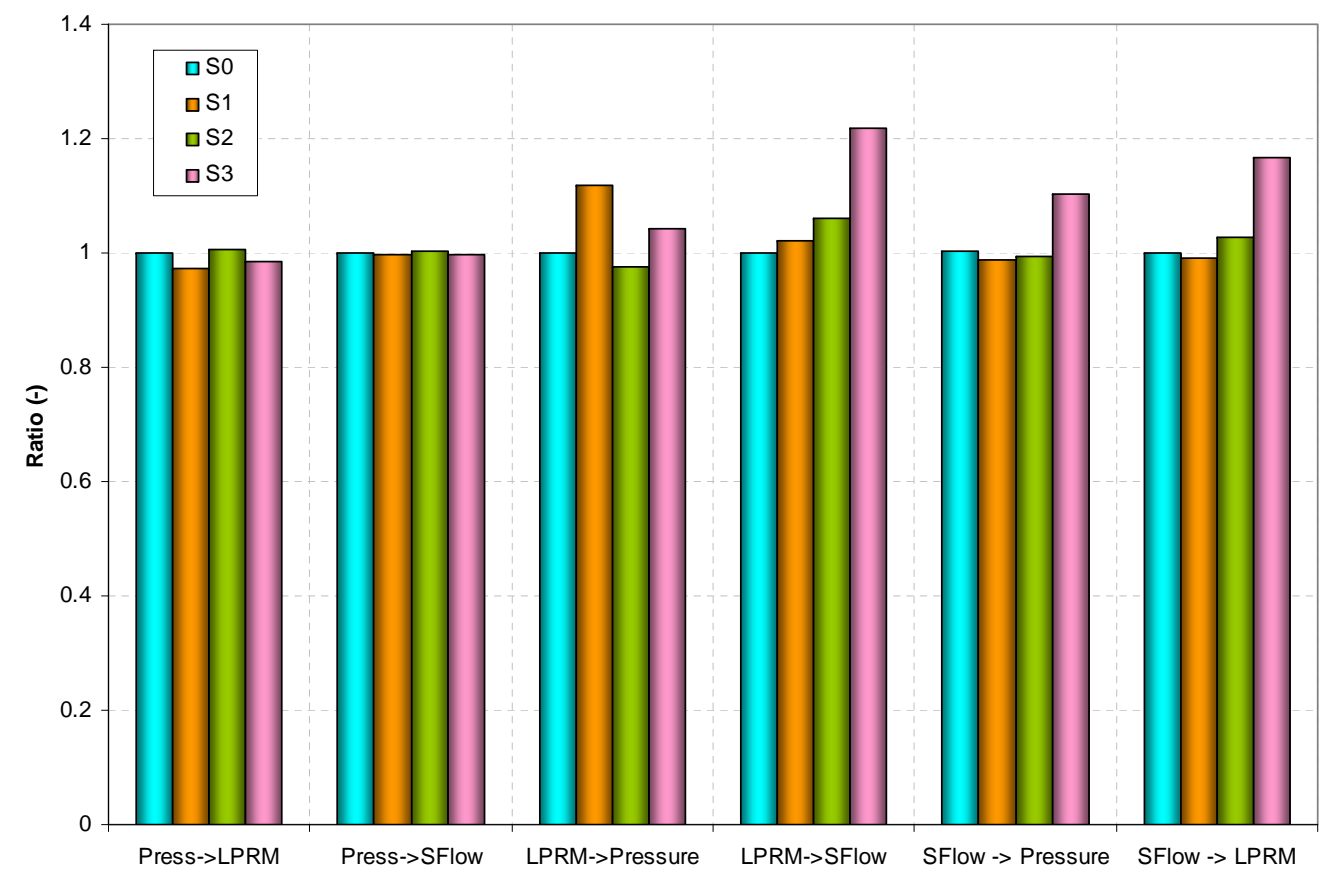

Figure 8: Ratio of PRESS, LPRM, SFLOW Interaction Terms between 4- and 3dimensional CVARMA Models.

estimated relationships between PRESS, LPRM, SFLOW, the ratios between the $4 \times 4$ sub-matrix and the initial $3 \times 3$ matrix were thus estimated as

$$
R t_{m}=\frac{S_{m, X->Y}^{\prime}}{S_{m, X->Y}}(m=0,1,2,3) .
$$

The results are shown in Fig. 8. Clearly, it can be seen that the ratios are in all case very close to 1 implying that enlarging the multi-variate system with another process signal stemming from the feedwater control system does not alter by any means the estimated relationships and trends. A certain sensitivity is seen for the higher-order matrices but this might be simply related to numerical convergence of the algorithm which deteriorates with increasingly higher-order matrices. 


\section{Benchmarking against STP method}

\subsection{Signal Transmission Path Analysis}

In studying causal relationships, signal transmission path analysis based on multivariate time series modeling has been widely used (Oguma, 1981, 1982a,b; Oguma and Turkan, 1985; Oguma, 1996). In addition, using this analysis allows detecting the feedback effect among variables of interest and allowing information about how the noise power is propagating from one variable to another.

In the current study, the STP analysis is performed for pairs of variables of interest, using the main two functions, i.e., the coherence $(\mathrm{COH})$ and the noise power contribution (NPC). The coherence function is very important to be evaluated since it represents the correlation between two variables in frequency domain and allows characterizing the dynamic relationship between the measured variables. The NPC function is used to evaluate noise source power propagation from one variable to another, allowing determination of the causal relationship between the variables of interest. The definition of these two main functions are as follows (Oguma, 1981).

Consider a linear dynamic system with feedback effect between variables $X_{1}$ and $X_{2}$ that can be described as:

$$
\begin{gathered}
X_{1}(z)=G_{12}(z) X_{2}(z)+N_{1}(z), \\
X_{2}(z)=G_{21}(z) X_{1}(z)+N_{2}(z) .
\end{gathered}
$$

Where $G_{21}(z)$ and $G_{12}(z)$ are the open loop transfer functions from $X_{1}$ to $X_{2}$ and from $X_{2}$ to $X_{1}$, respectively. $N_{1}(z)$ and $N_{2}(z)$ are noise sources to $X_{1}$ and $X_{2}$, respectively, and they are assumed to be stationary random with zero mean and mutually independent.

The auto power spectral density (APSD) functions $P_{11}(\omega)$ and $P_{22}(\omega)$ for the corresponding variables and the cross power spectral density (CPSD) function $P_{12}(\omega)$ are given by:

$$
\begin{aligned}
& P_{11}(\omega)=\left(1 / \Delta t^{2}\right)\left(\left|G_{12}(\omega)\right|^{2} Q_{22}(\omega)+Q_{11}(\omega)\right), \\
& P_{22}(\omega)=\left({ }^{1} / \Delta t^{2}\right)\left(\left|G_{21}(\omega)\right|^{2} Q_{11}(\omega)+Q_{22}(\omega)\right), \\
& P_{12}(\omega)=\left({ }^{1} / \Delta t^{2}\right)\left(G_{21}^{*}(\omega) Q_{11}(\omega)+G_{12}(\omega) Q_{22}(\omega)\right),
\end{aligned}
$$

where $\Delta=\left|1-G_{12}(\omega) G_{21}(\omega)\right|, Q_{11}(\omega)$ and $Q_{22}(\omega)$ are the APSDs of the respective noise sources, $|\cdot|$ denotes the absolute value and the superscript 
* the complex conjugate. Using Eq. (22)-(24), we define the COH function $\gamma_{12}^{2}(\omega)$ between $X_{1}$ and $X_{2}$ as

$$
\gamma_{12}^{2}(\omega)=\frac{\left|P_{12}(\omega)\right|^{2}}{P_{11}(\omega) P_{22}(\omega)} .
$$

The NPC ratio from $X_{2}$ to $X_{1}$ through the noise source $N_{2}$ is

$$
\Gamma_{12}(\omega)=\frac{\left|G_{12}(\omega)\right|^{2} Q_{22}(\omega)}{\left|G_{12}(\omega)\right|^{2} Q_{22}(\omega)+Q_{11}(\omega)} .
$$

Similarly, the NPC ratio from $X_{1}$ to $X_{2}$ through the noise source $N_{1}$ is

$$
\Gamma_{21}(\omega)=\frac{\left|G_{21}(\omega)\right|^{2} Q_{11}(\omega)}{\left|G_{21}(\omega)\right|^{2} Q_{11}(\omega)+Q_{22}(\omega)} .
$$

Note that the mutual comparison of $\mathrm{COH}$ and NPC functions allows the identification of causal relationship and the detection of the feedback between the two variables. Hence the following possibilities may exist Oguma (1996):

- The two functions are zero at all frequencies if no dynamic relationship between the two variables exists.

- If there is an explicit causal relationship only from $X_{1}$ to $X_{2}$, i.e., no feedback, then the NPC function from $X_{1}$ to $X_{2}$ coincide with the $\mathrm{COH}$ function, while the NPC function from $X_{2}$ to $X_{1}$ is zero at all frequencies, and vice versa.

- The two functions differ from each other if there is a feedback between the two variables.

It should be emphasized that the current STP analysis is carried out based on the VARMA modeling technique using the NAG subroutine g13dc (NAG, 2011). Under the condition of independent noise sources between the pair variables, the transfer functions and the power spectra of the noise sources are derived in terms of the VARMA model parameters. Because STP analysis is based on non-canonical VARMA model, its results can have some discrepancy with the causal structure of the actual nuclear plant. 


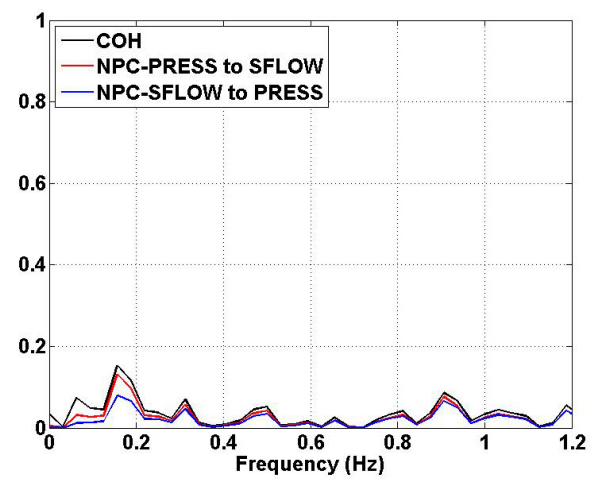

(a)

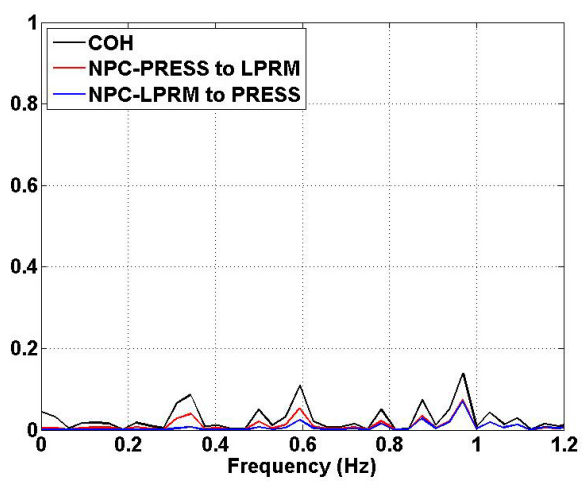

(b)

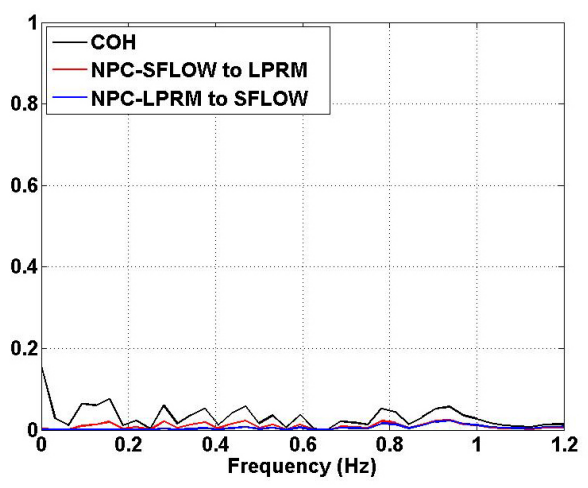

(c)

Figure 9: Application of STP to KKL Plant Instability Event Period 1.

\subsection{Application to KKL plant instability event}

Similar to CSARMA, the STP method is applied to each of the three periods, i.e., 1, 4 and 6 . The results for periods 1 and 6 are similar, therefore, for shortness we show only the one for period 1 in Fig.9. The results for period 4 are shown in Fig.10. We see that for period 1 and 6 , the $\mathrm{COH}$ and NPC functions for all signal pairs are almost less than 0.1 , which is negligible. This means the STP method does not show any intense dynamic interaction among all the three variables, i.e., SFLOW, PRESS and LPRM. However, for period 4, as expected (see section 2.2), a clear peak at around $0.9 \mathrm{~Hz}$ is observed for the $\mathrm{COH}$ function of all the signal pairs with a maximum value for the pair (LPRM, SFLOW), indicating a $\mathrm{COH}$ between the neutron 


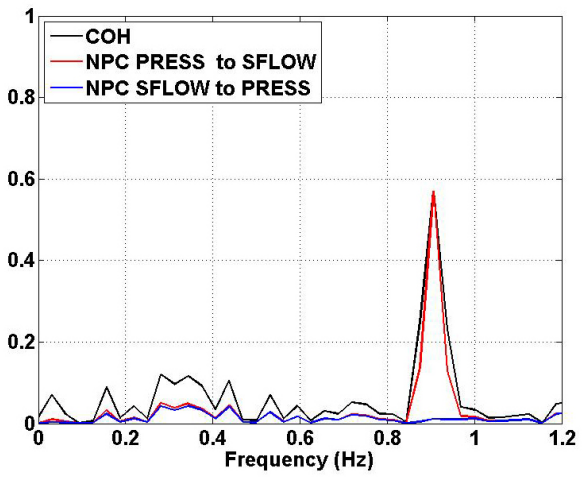

(a)

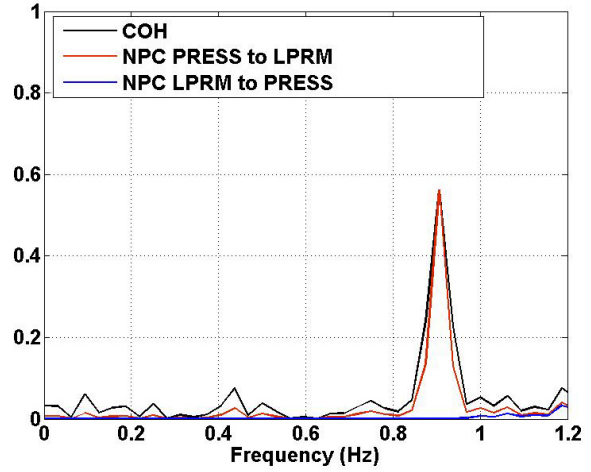

(b)

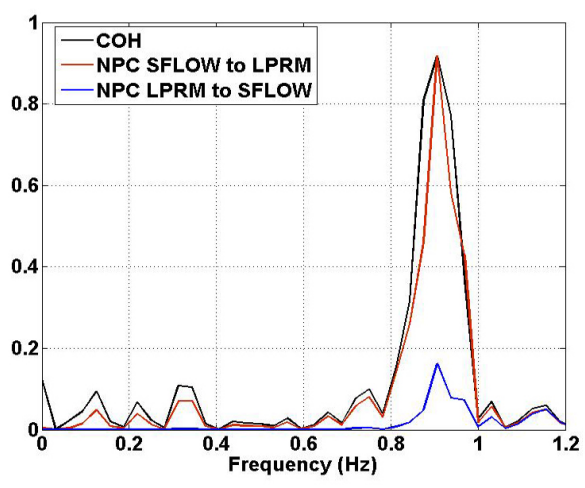

(c)

Figure 10: Application of STP to KKL Plant Instability Event Period 4.

flux and the steam flow at $0.9 \mathrm{~Hz}$. As can be seen from Fig.10, the NPC from reactor pressure to steam flow and neutron flux is dominant, while the NPC in the opposite direction, i.e., from neutron flux to reactor pressure and from steam flow to reactor pressure, is negligible. In addition, the NPC from steam flow to neutron flux is dominant while it is negligible in the opposite direction.

\section{Discussion}

Here, we note that the STP method failed to indicate significant effects among variables for periods 1 and 6 . However, the STP results for period 4 are in total agreement with those obtained using CSARMA approach. 
Also, the CSARMA method results for periods 1 and 6 indicated several dominant effects. The dominance of $\{$ SFLOW $\Longrightarrow$ PRESS $\}$ reflects correctly the "reactor master-turbine slave" concept with the pressure controller adjusting the opening of the turbine inlet/bypass valves to regulate the reactor pressure. The $\{\mathrm{LPRM} \Longrightarrow$ PRESS $\}$ dominance also follows from the same concept, i.e., during normal operation without any ex-vessel or system induced disturbances, the reactor pressure will be held constant and will increase/decrease as a response to a sudden core power (LPRM) increase/decrease. Hence, in this mode, the neutron flux will guide the reactor pressure evolution through the pressure controller, also guide the steam flow behavior as reflected by the observed $\{\mathrm{LPRM} \Longrightarrow$ SFLOW $\}$ dominance.

For period 4, both CSARMA and STP analysis methods indicated the opposite behavior of the influence from reactor pressure to steam flow which is inconsistent with the conventional "reactor master-turbine slave" concept. Thus, we can assume that reactor pressure is driven by some mechanisms which are not included into the "reactor master-turbine slave" concept. This would be in-line with the expected physics of the origin of the event, e.g., a fluctuation at the turbine/bypass valve inlet zone that propagated back to the vessel, inducing disturbances in the pressure control system.

\section{Conclusions}

This paper has presented a first application of a novel Continuous and Structural Autoregressive Moving Average (CSARMA) modeling approach to BWR noise analysis. The concept of this new method developed at Osaka University is to derive canonical models of a given multi-variate system in order to achieve a unique representation of the system dynamics. Its advantage is to provide more robust and reliable models as basis for signal analysis in general and for reactor diagnostics or causality analysis in particular. As a first step towards assessing the potential of CSARMA approach, the latter type of applications, a stability event that occurred in a Swiss BWR plant during power ascension phase, was analyzed. To that aim, CVARMA models for a multi-variate system comprising steam flow, reactor pressure and LPRM were estimated based on measurements taken during the closed to instability event. Similarly, corresponding CVARMA models were also estimated for more stable time periods that preceded and followed the event.

On that basis and focusing only on qualitative results at this stage, the estimated time-domain based CVARMA matrix structures were studied as 
function of the model order as well as between the various selected time periods. As main observations, it was found that the CVARMA matrix structures would clearly indicate a different dynamical state during the less stable period 4 compared to the two more stable periods 1 and 6 . Moreover, during the period 4, the CSARMA results would show a distinctly dominant relationship from steam flow to neutron flux and not vice versa, indicating thus that the core instability was caused by a plant system disturbance rather than the opposite. Furthermore, a very dominant relationship from reactor pressure to steam flow was indicated, this being in total opposition to the normal situation during reactor master/turbine slave operation when reactor pressure is adjusted through the steam flow. Hence, these CSARMA results can be interpreted as pointing out a disturbance in the pressure control system as primary cause for the instabilities during period 4 .

To benchmark all these findings, the frequency-domain based Signal Transmission Path (STP) method, currently being implemented at PSI as part of the overall noise analysis methodology for the Swiss reactors, was also applied. With the STP method, for period 4 exactly the same relationships as mentioned above were obtained. On the one hand, this consistency between both methods could be considered as having confirmed that the period 4 instabilities were caused by a pressure control system disturbance and not induced by the core. On the other hand, this consistency can also be considered as a verification of the CSARMA ability to correctly indicate the causality relationships even during less stable event.

It is also worth to note that for periods 1 and 6 the STP analysis failed to catch the dynamical relations between variables. The possible reason for it might be that the STP analysis is based on non-canonical VARMA model, therefore, its results can have some discrepancy with the causal structure of the actual nuclear plant. Also, the STP analysis is usually unable to estimate the interactions among variables for the cases when the system has weak noise function, e.g., very stable cases. However, the CSARMA modeling approach clearly indicated intense effects from both steam flow and neutron flux to reactor pressure for periods 1 and 6 . These results fully consistent with the normal dynamics of the plant.

From this point of view, further work will now focus on more detailed investigations of the CSARMA results at a quantitative level. As next step, conversion of the CSARMA method to the frequency domains will be launched and the verification analyses will be enlarged to other applications. 


\section{Acknowledgments}

This work was conducted as a collaboration between Osaka, KKL and PSI within the framework of the STARS project (http://stars.web.psi.ch). The authors would in this context like to acknowledge Dr. K. Sekhri from KKL for having provided additional support in relation to information and data on the KKL plant.

This work was partially supported by Minato Erato Project of Japan Science and Technology Agency (JST) and Grants-in-Aid for Scientific Research

\#25240036 and \#26540116 of Japan Society for the Promotion of Science (JSPS).

\section{References}

Brockwell, P., Davis, R., 1991. Time Series: Theory and Methods. Second edition ed., Springer, New York.

Demeshko, M., Washio, T., Kawahara, Y., 2013. A novel structural AR modeling approach for a continuous time linear markov system. ICDM workshop proceedings .

Demeshko, M., Washio, T., Kawahara, Y., Shimizu, S., 2014. A novel structural ARMA modeling across continuous and discrete time domains. In press .

Dokhane, A., Ferroukhi, H., Zimmermann, M., Aguirre, C., 2006. Spatial and Model-order Based Reactor Signal Analysis Methodology for BWR Core Stability Evaluation. Annals of Nuclear Energy 33, 1329-1338.

Ferroukhi, H., 2008. Reactor Signal Analysis of KKL BOC24 Data Measured on the 22.08.2007. Technical Report. PSI.

Fry, D., Leuba, J.M., Seeney, F., 1984. Use of Neutron Noise for Diagnostics of In-Vessel Anaomlaies in Light Water Reactors. Technical Report. ORNL Report TM-8774.

Gottschalk, J., 2001. An introduction into the SVAR methodology: Identification, interpretation and limitations of SVAR models. 
Hinrichsen, D., Pritchard, A., 2005. Mathematical Systems Theory I, Modelling, State Space Analysis, Stability and Robustness. volume 48. Springer.

Kanemoto, S., Andoh, Y., Yamamoto, F., Kitamoto, K., Nunome, K., 1982. Identification of Pressure Control System Dynamics in BWR Plant by Multivariate Autoregressive Modeling Technique. Journal of Nuclear Science and Technology 19, 58-68.

Kawahara, Y., Shimizu, S., Washio, T., 2011. Analyzing relationships between ARMA processes based on non-gaussianity of external influences. Neurocomputing 74, 2212-2221.

Kwatny, H., Fink, L., 1975. Acoustics, stability, and compensation in boiling water reactor pressure control systems. IEEE Trans. Autm. Control 1, 727-739.

Levy, H., Lessman, F., 1992. Finite Difference Equations. Dover.

Mainassara, Y.B., Francq, C., 2009. Estimating structural VARMA models with uncorrelated but non-independent error terms. Journal of Multivariate Analysis 102, 496-505.

Moneta, A., Entner, D., Hoyer, P., Coad, A., 2010. Causal Inference by Independent Component Analysis with Applications to Micro- and Macroeconomic Data. Jena Economic Research Papers , 3-4.

NAG, 2011. NAG Toolbox for Matlab g13dc. The Numerical Algorithms Group Ltd, Oxford, UK.

Oguma, R., 1981. Method to evaluate signal transmission paths in dynamic systems and its application to reactor noise analysis. J. Nucl. Sc. Tech 18, 835-844.

Oguma, R., 1982a. Cohenerence analysis of systems with feedback and its application to bwr noise investigation. Prog. Nucl. Energy 9, 137-148.

Oguma, R., 1982b. Extended partial and multiple coherence analyses and their application to reactor noise inves-tigation. J. Nucl. Sc. Tech. 19, $543-554$. 
Oguma, R., 1996. Investigation of power oscillation mechanisms based on noise analysis at forsmark-1 bwr. Ann. Nucl. Energy 23, 469-485.

Oguma, R., Turkan, E., 1985. Application of an improved multivariable noise analysis method to investigation of PWR noise; signal transmission path analysis. Progress in Nuclear Energy 15, 863-873.

Pazsit, I., 1999. Development of core diagnostic methods and their application at swedish bwrs and pwrs. Jour. Nucl. Sc.Tech. 36, 473-485.

Rotander, C., 1999. Methods to Identify Stability properties for a Thermaohydraulic Channel and from Reactor Noise Measurements. Ph.D. thesis. Royal Institute of Technology. Sweden.

Shea, B.L., 1987. Estimation of multivariate time series. Journal of Time Series Analysis 8, 95-110.

Stamer, O., Tweedie, R., Brockwell, P., 1996. Existence and stability of continuous time threshold ARMA process. Statistica Sinica 6, 715-732.

Sweeney, F., 1987. Utility Guidelines for Reactor noise Analysis. Technical Report NP-4970. EPRI.

Williams, M., 1977. Reactor noise - SMORN II. Progress in Nucl. Energy 1, 73-804.

Wilson, D.H., 2006. Identifying potential interaction between bwr plant dynamics and power system electromechanical oscillations.

Zylbersztejn, F., Tran, H., Pazsit, I., Demaziere, C., Nylen, H., 2013. On the dependence of the noise amplitude on the correlation length of inlet temperature fluctuations in PWRs. Ann. Of. Nucl. Energy 57, 134-141.

\section{Appendix A. Illustrative example of CSARMA application}

Dynamics of a simple coupled oscillator depicted depicted in Fig. A.11. Its dynamics is represented by the following two differential equations.

$$
\begin{aligned}
& \ddot{x}_{1}=\frac{-2 \kappa x_{1}}{M}-\frac{c}{M} v_{1}, \\
& \ddot{x}_{2}=\frac{-2 \kappa x_{2}}{M}-\frac{c}{M} v_{2} .
\end{aligned}
$$




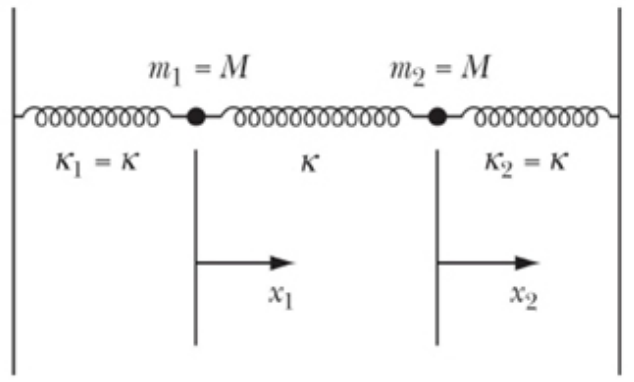

Figure A.11: Coupled oscillators.

The mass of each object is $M$, and the spring constant of each spring is $\kappa$ where their right most and left most ends are fixed at the two walls. Two state variables $x_{1}$ and $x_{2}$ represent the deviations of the mass positions from their equilibrium, and $v_{1}=\dot{x}_{1}$ and $v_{2}=\dot{x}_{2}$ are their velocities. We also assume some damping forces acting on the masses caused by air friction where their coefficient is $c$.

The two deviations $x_{1}$ and $x_{2}$ are observed by a photo camera in our problem. The camera has an exposure time $\Delta t$. This temporal ambiguity creates observation errors of $x_{1}$ and $x_{2}$, since the objects move over the exposure time. As the result, the camera observes their average deviations during $[t, t+\Delta t]$. Accordingly, their errors from the exact deviations is $\frac{\Delta t}{2} v_{1}$ for the object 1 and $\frac{\Delta t}{2} v_{2}$ for the object 2 .

This system is exactly represented by a controllable canonical form of the state space model in continuous time domain which explicitly indicates kinematics, air friction and observation errors. It consists of the linear differential system equations having external process noises and observation equations of the state variables (Hinrichsen and Pritchard, 2005). Each of the linear differential system equations represents an individual mechanism independently disturbed by the external noise.

$$
\begin{aligned}
\frac{d X}{d t} & =\left[\begin{array}{cccc}
0 & 0 & 1 & 0 \\
0 & 0 & 0 & 1 \\
\frac{-2 \kappa}{M} & \frac{\kappa}{M} & -\frac{c}{M} & 0 \\
\frac{\kappa}{M} & \frac{-2 \kappa}{M} & 0 & -\frac{c}{M}
\end{array}\right] X+\left[\begin{array}{ll}
0 & 0 \\
0 & 0 \\
1 & 0 \\
0 & 1
\end{array}\right] W \text { and } \\
Y & =\left[\begin{array}{cccc}
1 & 0 & \frac{\Delta t}{2} & 0 \\
0 & 1 & 0 & \frac{\Delta t}{2}
\end{array}\right]^{T} X,
\end{aligned}
$$


where $X=\left[x_{1}, x_{2}, v_{1}, v_{2}\right]^{T}$ and $W=\left[w_{1}, w_{2}\right]^{T} . w_{1}$ and $w_{1}$ are the external process noises of $x_{1}$ and $x_{2}$. The controllable canonical form of the state space model has direct one to one correspondence to the CVARMA model (Hinrichsen and Pritchard, 2005; Brockwell and Davis, 1991), and in the case of the coupled oscillator, we have the following CVARMA $(2,1)$ model.

$$
\begin{aligned}
Y^{(2)}(t)= & {\left[\begin{array}{cc}
\frac{-2 \kappa}{M} & \frac{\kappa}{M} \\
\frac{\kappa}{M} & \frac{-2 \kappa}{M}
\end{array}\right] Y^{(0)}(t)+\left[\begin{array}{cc}
-\frac{c}{M} & 0 \\
0 & -\frac{c}{M}
\end{array}\right] Y^{(1)}(t) } \\
& +W^{0}(t)+\left[\begin{array}{cc}
\frac{\Delta t}{2} & 0 \\
0 & \frac{\Delta t}{2}
\end{array}\right] W^{(1)}(t)
\end{aligned}
$$

We gave the values of the spring constant $\kappa=0.38 \mathrm{~N} / \mathrm{m}$, the mass $\mathrm{M}=1 \mathrm{~kg}$, the air resistance coefficient for the mass $c=0.5 \mathrm{Ns} / \mathrm{m}$ and the exposure time $\Delta t=1 \mathrm{~s}$, the period of oscillation $T=2 \pi \sqrt{\frac{M}{\kappa}}=10.2 \mathrm{~s}$. Then we transformed this CVARMA model to its corresponding SVARMA and VARMA models by using Eq.(6), (7) and (8) and the inversed relations of Eq.(12) and (13) under a time granularity $\delta t=0.1 \mathrm{~s}$ far smaller than $\Delta t$ to simulate an approximately continuous process. We confirmed that the stationarity, the controllability and the observability of the VARMA model in this discrete time domain with $\delta t$ hold to ensure the conditions required for the VARMA modeling. We further generated a time series $Y(t)$ by using the derived VARMA model parameters and Eq.(1). The length of the generated time series is 1000 data points. The external bivariate noises $U(t)$ were generated by using an i.i.d. $N\left(0, \sigma^{2}\right)$ distribution where $\sigma$ is randomly chosen from $[0.3,0.7]$ to maintain the identifiability of the VARMA model. Since the sampling time of the system is $\Delta t=1 \mathrm{~s}$, we further sampled the generated time series by choosing every 10th point in it.

We applied the CSARMA algorithm shown in Fig. 5 to this sampled time series and estimated the VARMA, SVARMA and CVARMA models. The correct models orders, $\mathrm{p}=2$ and $\mathrm{q}=1$, are provided for the estimation. Table A.2 shows the comparison between the original models derived from the CVARMA model under the discretization with $\Delta t=1 \mathrm{~s}$ and the estimated models. The table indicates that the SVARMA and CVARMA models estimated by the CSARMA approach match well with their original models. We also see that the original VARMA model matches well with the VARMA model estimated by Maximum-Likelihood method. The last corresponds to our conclusion in the previous section that the objective system is represented by a unique VARMA model when it is linear Markov, stationary, 
Table A.2: The parameter matrices of the original and estimated VARMA, SVARMA and CVARMA models, where $Z$ and $\hat{Z}$ represent original and estimated matrices, respectively, for $Z=\Phi, \Theta, \Psi, \Omega, S$ and $R$.

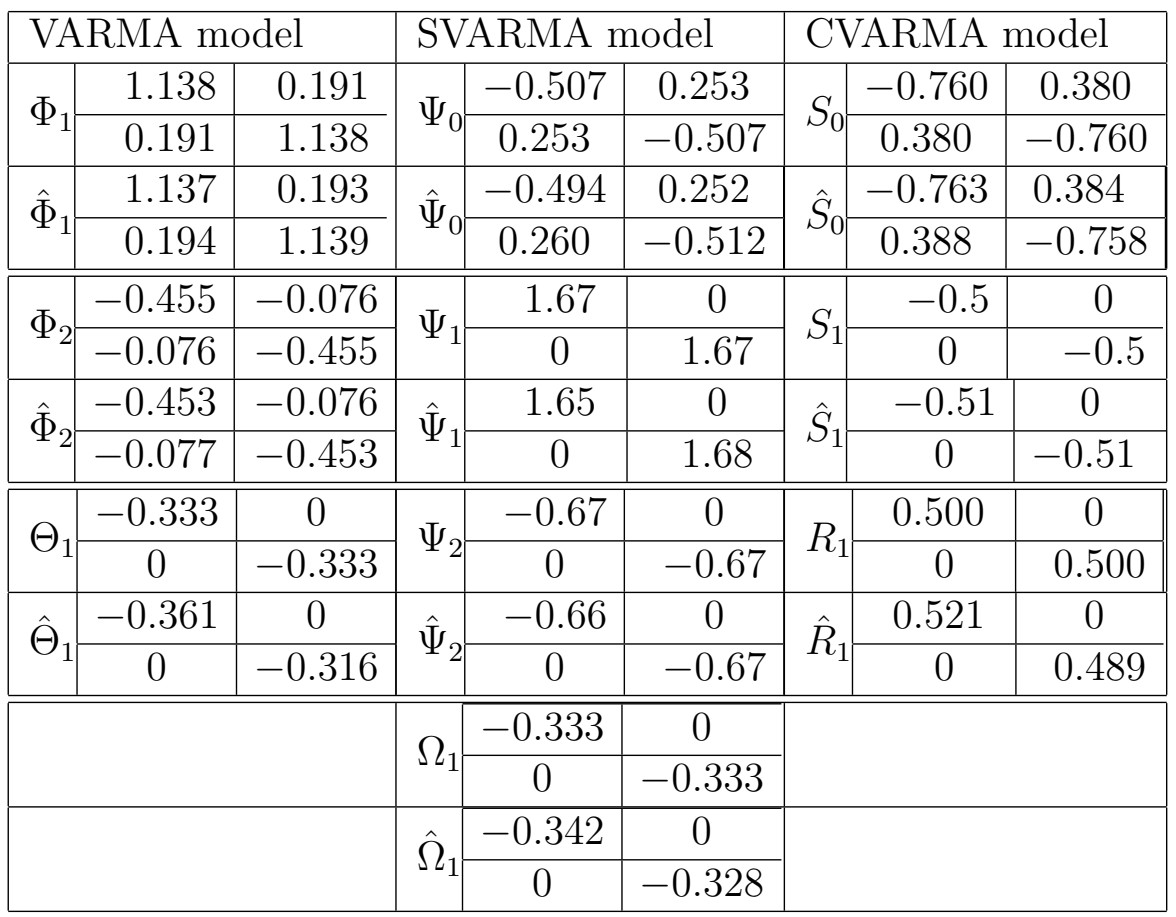

controllable and observable system in continuous time domain. Accordingly, the CSARMA modeling appropriately reconstructs the original SVARMA and CVARMA models of the system from a given time series, and provides the correct canonical relation between the variables in the original system. 